\title{
Verbessertes PFS bei Brustkrebs
}

Die zusätzliche Gabe des monoklonalen Antikörpers Denosumab zur adjuvanten Therapie mit Aromatasehemmern bei postmenopausalen, hormonrezeptorpositiven Brustkrebspatientinnen reduziert die Rückfallsrate um 18\%. Das ist das zentrale Ergebnis der von der Austrian Breast \& Colorectal Cancer Study Group (ABCSG) geleiteten Brustkrebsstudie ABCSG 18 zum krankheitsfreien Überleben, die Ende vergangenen Jahres beim San Antonio Breast Cancer Symposium (SABCS) präsentiert wurde.

Zusätzlich zu der bereits Mitte des Jahres publizierten Reduktion von therapieinduzierter Osteoporose und Knochenbrüchen durch die zusätzlich Verabreichung von Denosumab zur endokrinen Adjuvanstherapie mit Aromatase-Inhibitoren, konnte mit den nun publizierten Ergebnissen ein weiterer Vorteil dieser Therapie gezeigt werden. Die Auswirkungen von Denosumab auf die Knochengesundheit hatten den ersten Studienendpunkt dargestellt, der durch eine Reduktion von therapieinduzierter Osteoporose und Knochenbrüchen ohne zusätzliche Toxizität um $50 \%$ erreicht wurde.

\section{Auswirkungen \\ auf die klinische Praxis}

Der Einfluss von Denosumab auf das krankheitsfreie Überleben (disease-free survival, DFS) bildete einen weiteren Studienendpunkt. Insgesamt wurden 370 DFS-Ereignisse im Zeitraum von vier Jahren dokumentiert, davon 203 in der Placebo-Gruppe und 167 im Denosumab-Arm. Diese Reduktion der Rezidivrate von Brustkrebs ist knapp an der statistischen Signifikanzgrenze $(H R=0,816, p=0,051)$.

„Dieses Ergebnis ist hoch erfreulich, zeigt es doch, dass adjuvantes Denosumab nicht nur Knochenbrüche halbiert, sondern auch die Rezidivrate von Brustkrebs verringert", stellt Univ.-Prof. Dr. Michael Gnant, Leiter der Universitätsklinik für Chirurgie der MedUni Wien, stellvertretender Leiter des Comprehensive Cancer Center (CCC) und Präsident der ABCSG, fest: „Wir haben immer davon geträumt, über eine Veränderung des Microen- vironments die Heilungsrate günstig zu beeinflussen, und mit ABCSG 18 gelingt uns das ein weiteres Mal."

Explorative Subgruppenanalysen zeigen, dass einige Patientinnen besonders deutlich von der praktisch nebenwirkungsfreien Zugabe von Denosumab zur üblichen AromataseInhibitor-Therapie profitieren: Besonders bei Tumoren über zwei Zentimeter Größe und frühzeitigem Behandlungsbeginn sowie bei Tumoren mit besonders hoher Rezeptordichte zeigt sich ein klar signifikantes Ergebnis.

Die Ergebnisse werden, so Gnant, bald Änderungen in der klinischen Praxis zur Folge haben: „Damit steht Denosumab, das kaum Nebenwirkungen aufweist, in der Adjuvansthera-

pie insgesamt über den Bisphosphonaten und sollte meiner Meinung nach allen postmenopausalen, hormonrezeptorpositiven Brustkrebspatientinnen angeboten werden." Von Bisphosphonaten, die in der Osteoporosetherapie eingesetzt werden, weiß man, dass sie positiven Einfluss auf das krankheitsfreie Überleben haben können. Denosumab wirkt ähnlich wie Bisphosphonate, hat bei stärkerer Wirksamkeit allerdings weniger Toxizität und kann sehr einfach subkutan verabreicht werden.

Wien klin Mag 2016 · 19:21

DOI 10.1007/s00740-016-0091-8

Online publiziert: 24. Januar 2016

C) Springer-Verlag Wien 2016

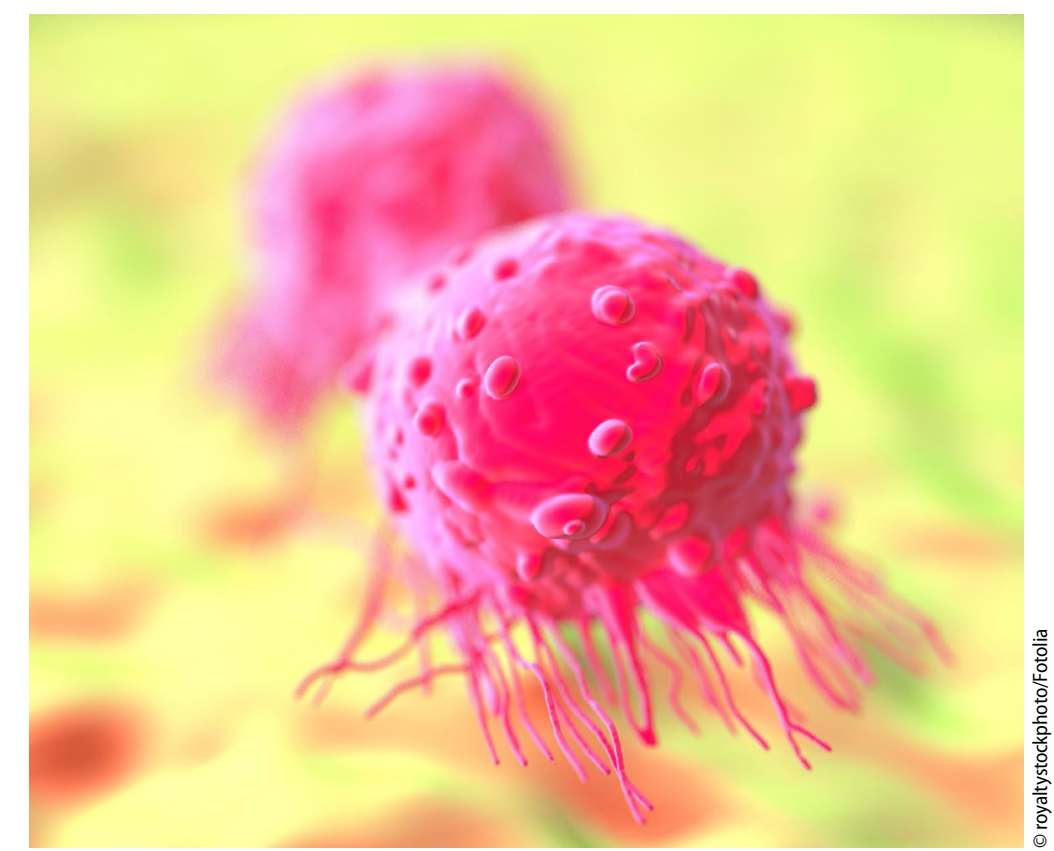

$\Delta$ Adjuvantes Denosumab halbiert das Auftreten von Knochenbrüchen und verringert die Rezidivrate von Brustkrebs
Quelle: Presseaussendung Austrian Breast \& Colorectal Cancer Study Group \& MedUni Wien 